FERMILAB-TM-2043

\title{
An Insertion to Eliminate Horizontal Temperature of High Energy Electron Beam
}

\author{
A.V. Burov and V.V. Danilov \\ Fermi National Accelerator Laboratory \\ P.O. Box 500, Batavia, Illinois 60510
}

March 1998 


\section{Disclaimer}

This report was prepared as an account of work sponsored by an agency of the United States Government. Neither the United States Government nor any agency thereof, nor any of their employees, makes any warranty, expressed or implied, or assumes any legal liability or responsibility for the accuracy, completeness, or usefulness of any information, apparatus, product, or process disclosed, or represents that its use would not infringe privately owned rights. Reference herein to any specific commercial product, process, or service by trade name, trademark, manufacturer, or otherwise, does not necessarily constitute or imply its endorsement, recommendation, or favoring by the United States Government or any agency thereof. The views and opinions of authors expressed herein do not necessarily state or reflect those of the United States Government or any agency thereof.

\section{Distribution}

Approved for public release; further dissemination unlimited. 


\title{
An Insertion to Eliminate Horizontal Temperature of High Energy Electron Beam
}

\author{
A. V. Burov * and V. V. Danilov* \\ Fermi National Accelerator Laboratory \\ P.O. Box 500, Batavia, Illinois 60510
}

March 16, 1998

\begin{abstract}
High energy electron cooling with a circulated electron bunch could significantly increase the luminosity of hadron colliders. One of the significant obstacles is high horizontal temperature of electron bunches, suppressing dramatically calculated cooling rates. Recently, a transformation of betatron coordinates and angles for elimination of the radial temperature was found. In our paper, we present a simple scheme to make up this transformation by thin quadruples, drifts and a solenoid.
\end{abstract}

\section{Introduction}

A possibility to use circulating electron bunches for electron cooling in hadron colliders is an attractive idea, meeting several obstacles for realization (see e. g. [1] with a vast list of references). One of the difficulties is that high radial temperature of the electron bunch in a storage ring would strongly suppress cooling rates. In electron storage rings, bunches are practically flat: their horizontal emittance is orders of magnitude higher than vertical one. A way to eliminate the horizontal temperature for such bunches was recently suggested in [2]. A matrix, which transforms the flat distribution into a distribution without transverse angles, was found there. It should be noted, that such a transformation does not contradict to the Liouville's theorem: the $4 \mathrm{D}$ transverse phase volume $\left(x x^{\prime} y y^{\prime}\right)$ for the flat bunch is zero, as well as for the bunch with zero angles. With this transformation, the calculated cooling rates are strongly shifted to a practically interesting range of values.

In the following chapter, this transformation is discussed. Then, its practical realization on the base of thin quadruples, drifts and a solenoid is considered.

\footnotetext{
* On leave from Budker INP, 630090, Novosibirsk, Russia.
} 


\section{Transformation Matrix}

Let us consider a distribution where vertical coordinates and angles are connected in a particular way with the horizontal ones:

$$
\left[\begin{array}{c}
y \\
y^{\prime}
\end{array}\right]=\left[\begin{array}{cc}
0 & -\beta \\
1 / \beta & 0
\end{array}\right]\left[\begin{array}{c}
x \\
x^{\prime}
\end{array}\right] \equiv F \cdot\left[\begin{array}{c}
x \\
x^{\prime}
\end{array}\right],
$$

where $\beta$ is an arbitrary parameter; $x, x^{\prime}$ and $y, y^{\prime}$ stand for horizontal and vertical coordinates and angles correspondently. Note that $F^{2}=-I$, where $I$ is an unity matrix. With this condition, the distribution is vortex. The $2 \mathrm{D}$ velocity is transverse to radius-vector and their values are proportional to each other:

$$
x x^{\prime}+y y^{\prime}=0, \quad x^{\prime 2}+y^{\prime 2}=\left(x^{2}+y^{2}\right) / \beta^{2} .
$$

In this case, the transverse velocity can be eliminated by solenoid with longitudinal field $B_{s}$, which is inversely proportional to parameter $\beta$ in (1):

$$
B_{s}=-2 p c / e \beta
$$

where $p$ is a momentum of particles, $c$ is a velocity of light, $e$ is a proton's charge. In the result, the particles have no angles inside this solenoid.

So, the problem reduces to building a transformation satisfying the relation (1). To start, an uncoupled 2D transformation can be introduced:

$$
U_{M N}=\left[\begin{array}{cc}
M & 0 \\
0 & N
\end{array}\right]
$$

where $M, N$ are $2 \times 2$ matrices of horizontal and vertical motion. Rotated on $45^{\circ}$, it transforms in a skew block:

$$
C=R_{4}^{-1}(\alpha) \cdot U_{M N} \cdot R_{4}(\alpha)=1 / 2\left[\begin{array}{cc}
M+N & M-N \\
M-N & M+N
\end{array}\right] .
$$

Here $R_{4}(\alpha)$ is a $4 \times 4$ matrix of rotation on the angle $\alpha=45^{\circ}$ :

$$
R_{4}(\alpha)=\left[\begin{array}{cc}
I \cos \alpha & I \sin \alpha \\
-I \sin \alpha & I \cos \alpha
\end{array}\right]=\frac{1}{\sqrt{2}}\left[\begin{array}{cc}
I & I \\
-I & I
\end{array}\right],
$$

with $I$ as a unit $2 \times 2$ matrix.

The inverse matrix to (5) is obtained by a substitution $M \leftrightarrow N$ :

$$
\begin{array}{r}
C^{-1}=R_{4}^{-1}(\alpha) \cdot U_{N M} \cdot R_{4}(\alpha)= \\
R_{4}(\alpha) \cdot U_{M N} \cdot R_{4}^{-1}(\alpha)=1 / 2\left[\begin{array}{cc}
N+M & N-M \\
N-M & M+N
\end{array}\right] .
\end{array}
$$


As it was noted, vertical coordinate and angle can be taken as zeroes at the entrance; so, the skew transformation results in:

$$
\left[\begin{array}{c}
x \\
x^{\prime}
\end{array}\right]=1 / 2[M+N]\left[\begin{array}{l}
x_{0} \\
x_{0}^{\prime}
\end{array}\right]
$$

and

$$
\left[\begin{array}{c}
y \\
y^{\prime}
\end{array}\right]=1 / 2[M-N]\left[\begin{array}{c}
x_{0} \\
x_{0}^{\prime}
\end{array}\right]
$$

where $x_{0}, x_{0}^{\prime}$ are horizontal coordinate and angle at the entrance of this transformation. It can be seen that the required condition (1) is satisfied when $M-N=F \cdot(M+N)$, which gives

$$
N=-F \cdot M
$$

Thus, any transformation (5) turns the flat distribution into the vortex one (2), as soon as the last condition (10) is satisfied.

If applied to the cooling purposes, a sequence of transformations for the electron bunch can be following [2]:

- the skew block $C$ transforms the flat distribution into the vortex one;

- the solenoid entrance kick eliminates electron velocity;

- a drift inside the solenoid needed for the cooling itself;

- the solenoid exit kick restores the vortex distribution;

- the inverse skew block $C^{-1}$ returns the flat distribution.

In the following section, it is shown how the required transformation can be constructed on the elementary base of thin lenses and drifts.

\section{Scheme with quadruples and drift spaces}

The horizontal matrix $M$ can be taken in the simplest form

$$
M=\left[\begin{array}{cc}
\cos (\mu) & \beta \sin (\mu) \\
-1 / \beta \sin (\mu) & \cos (\mu)
\end{array}\right],
$$

where $\beta$ is a parameter, that determines the magnetic field of the solenoid (3); a phase advance $\mu$ is a free parameter. The form of the vertical matrix $N$ is followed from (1):

$$
N=\left[\begin{array}{cc}
-\sin (\mu) & \beta \cos (\mu) \\
-1 / \beta \cos (\mu) & -\sin (\mu)
\end{array}\right]
$$




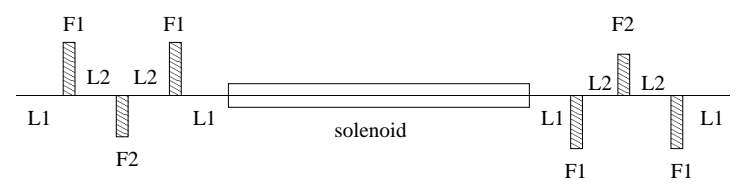

Figure 1: General scheme of the insertion.

For these matrices, the coordinate and velocity parts of the vortex transformation $C$ write:

$$
\begin{gathered}
{\left[\begin{array}{l}
x \\
y
\end{array}\right]=\frac{1}{\sqrt{2}} R_{2}(-\mu-\pi / 4)\left[\begin{array}{c}
x_{0} \\
-\beta x_{0}^{\prime}
\end{array}\right],} \\
{\left[\begin{array}{l}
x^{\prime} \\
y^{\prime}
\end{array}\right]=\frac{1}{\sqrt{2} \beta} R_{2}(-\mu-3 \pi / 4)\left[\begin{array}{c}
x_{0} \\
-\beta x_{0}^{\prime}
\end{array}\right],}
\end{gathered}
$$

$R_{2}(\alpha)$ is the rotation matrix $2 \times 2$ similar to (6):

$$
R_{2}(\alpha)=\left[\begin{array}{cc}
\cos \alpha & \sin \alpha \\
-\sin \alpha & \cos \alpha
\end{array}\right]
$$

The vortex distribution has a round shape when the beta-function at the entrance is equal to the vortex parameter $\beta$, and the derivative of the betafunction is zero. The radius of such round beam is $\sqrt{2}$ times smaller than the half of horizontal size of the input flat beam.

In Fig. 1 the general scheme of the insertion is shown. It consists of

- the three quads with drift spaces in between (symmetric triplet), twisted on the angle $45^{\circ}$ around the beam velocity direction;

- solenoid;

- the same triplet, twisted on opposite angle $-45^{\circ}$ for making the inverse transformation.

Note that the storage ring has no coupling due to this insertion. So, we have to create uncoupled betatron transformation with special relation 1 between the horizontal and vertical matrices.

The matrix $M$ is given by

$$
M=L(s 1) F(f 1) L(s 2) F(f 2) L(s 2) F(f 1) L(s 1),
$$

where $L$ is a matrix of drift space, $F$ is a matrix of a thin quadrupole, $s 1, s 2$ are lengths of the outer and the inner drift spaces; $f 1, f 2$ are focal lengths of the outer and the inner quads. The vertical matrix $N$ is given by the same formula but with opposite sign of quadrupole's strength:

$$
N=L(s 1) F(-f 1) L(s 2) F(-f 2) L(s 2) F(-f 1) L(s 1) .
$$




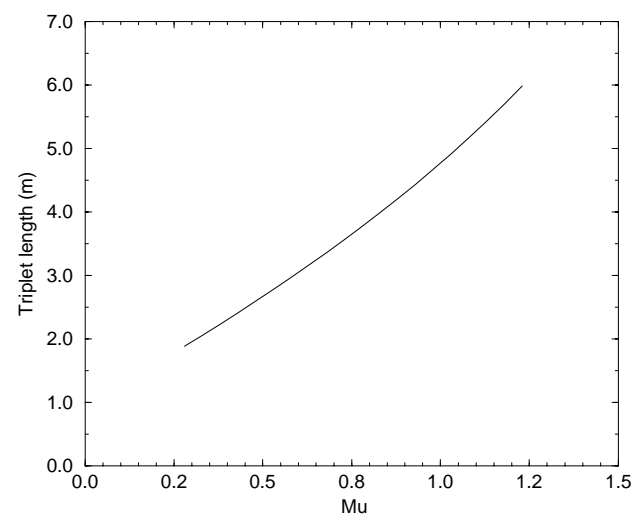

Figure 2: Length of the insertion vs. $\mu$.

The solution for $s 1, s 2$ and $f 1, f 2$ was found numerically as a function of the free parameter $\mu$. The solution exists for $.18<\mu<2.08$, it does not beyond this interval. The solution can be found for every parameter $\beta$ : it just gives a scale for length. All the parameters with the dimension of length are proportional to it, the magnetic field is inversely proportional.

The length of triplet versus parameter $\mu$ is presented in Fig.2. The most economical solution corresponds to small $\mu$. For $\mu=.38$, assuming the scale parameter $\beta=3.33 \mathrm{~m}$ and the energy of electron beam $E=500 \mathrm{MeV}$, the triplet parameters are found: $s 1=4.5 \mathrm{~cm}, s 2=107.2 \mathrm{~cm}$, longitudinal field $B_{s}=10.29 \mathrm{KGs}$, and the focal lengths of two lenses are $-1.373 \mathrm{~m}$ and 1.172 $\mathrm{m}$. For $10 \mathrm{~cm}$ of the quadruple length, this corresponds to $-1.214 \mathrm{KGs} / \mathrm{cm}$ and $1.423 \mathrm{KGs} / \mathrm{cm}$ of the field gradient inside the quadruples. The total length of the triplet is about $2 \mathrm{~m}$, that looks reasonable for insertions of such a kind.

The beam transformation inside the triplet is illustrated in Fig. (3), kindly presented to the authors by A. Sery. Vectors show the velocities $\left(x^{\prime}, y^{\prime}\right)$ for some random coordinates $(x, y)$. The coordinates and angles are normalized by $\beta$.

\section{Conclusion}

It was proved, that the transformation for a radial temperature elimination can be made by a triplet with reasonable parameters. In fact, this plane-vortex transformation of the electron distributions can be considered as an instrument of a general accelerator usage. 

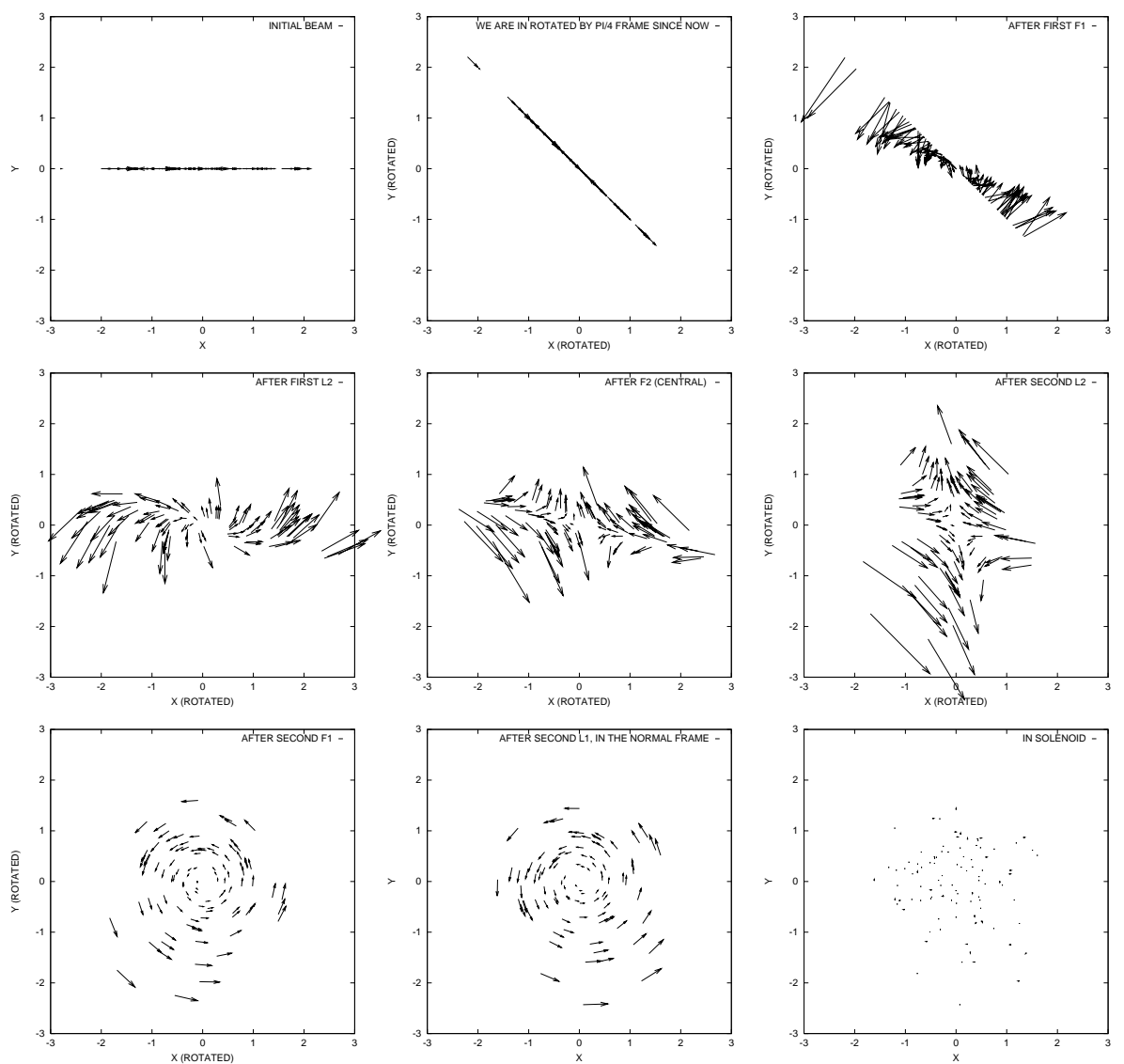

Figure 3: Distribution transformation inside the triplet. Vectors show normalized velocities $\left(x^{\prime}, y^{\prime}\right) / \beta$ for some random coordinates $(x, y)$ (presented by A. Sery). 


\section{Acknowledgments}

The authors are grateful to Ya. Derbenev for comments on his papers. They acknowledge encouraging discussions with P. Colestock, S. Y. Lee and V. Parkhomchuk. Special thanks to A. Sery for the suggested illustration and the manuscript corrections and to V. Shiltsev for useful remarks.

\section{References}

[1] S.Y.Lee, P.Colestock, and K.Y.Ng, "Electron Cooling in High Energy Colliders", FERMILAB-FN-657,(1997).

[2] Ya. Derbenev, " Adapting Optics for High Energy Electron Cooling", University of Michigan Preprint,February, 1998. 